\title{
Anti-CD3 OKT3/Humanized Anti-GD2 3F8 Bispecific Antibody-activated T Lymphocytes
}

National Cancer Institute

\section{Source}

National Cancer Institute. Anti-CD3 OKT3/Humanized Anti-GD2 3F8 Bispecific Antibody-

activated T Lymphocytes. NCI Thesaurus. Code C116330.

Autologous activated T cells that have been coated with bispecific antibodies (BiAb)

comprised of anti-CD3 murine monoclonal antibody OKT3 heteroconjug ated to anti-GD2 humanized monoclonal antibody 3F8 (hu3F8), with potential antineoplastic and immunomodulating activities. In vitro, T cells are exposed to OKT3, which binds to the T cell receptor-CD3 complex on the T cell surface, crosslinks the CD3 receptors and leads to T cell activation. In turn, the hu3F8 monoclonal antibody is heteroconjugated to OKT3. Upon administration, anti-CD3 $x$ anti-GD2 bispecific antibody-armed activated T cells attach to GD2-expressing tumor cells, thereby selectively cross-linking T cells and tumor cells. This results in selective cytotoxicity towards the GD2-expressing tumor cells. In addition, cytokine and chemokine secretion by the $T$ cells further activates the immune system, which leads to the recruitment and activation of cytotoxic T lymphocytes (CT Ls), and additional CT L-mediated tumor-specific cell lysis. GD2, a disialogang lioside and tumor-associated antigen, is overexpressed in a variety of tumor cell types. CD3 is part of the functional T cell receptor (TCR) complex, which is necessary for antigen recognition by $T$ cells, and is required for signal transduction. 